\title{
Strength Loss Counteracts Age-related Improvements in Muscle Fatigue Properties when Considering Functional Task Endurance-A Modeling Study
}

\author{
Avin Keith $\mathbf{G}^{3}$, Tumuluri Aruna ${ }^{4}$, Looft John MSE $^{2}$ and Frey-Law Laura $\mathbf{A}^{1,2^{*}}$ \\ ${ }^{1}$ Department of Physical Therapy and Rehabilitation Science, Carver College of Medicine, University of lowa, Iowa City, IA, USA \\ ${ }^{2}$ Virtual Soldier Research Team, Center for Computer-Aided Design, College of Engineering, University of lowa, Iowa City, IA, USA \\ ${ }^{3}$ Center for Translational Musculoskeletal Research, School of Health and Rehabilitation Sciences, Indiana University, Indianapolis, IN, USA \\ ${ }^{4}$ Dassault Systems SIMULIA Corp., 166 Valley St., Providence, RI, USA
}

*Corresponding author: Laura Frey-Law, PhD, PT, Associate Professor, Department of Physical Therapy and Rehabilitation, Science, 1-252 Medical Education Bldg, The University of lowa, lowa City, IA 52242, USA, Tel: 319-335-9804; E-mail: laura-freylaw@uiowa.edu

Rec date: Apr 14, 2014; Acc date: June 27, 2015; Pub date: June 29, 2015

Copyright: () 2015 Avin KG, et al. This is an open-access article distributed under the terms of the Creative Commons Attribution License, which permits unrestricted use, distribution, and reproduction in any medium, provided the original author and source are credited.

\begin{abstract}
Background: Older adults may fatigue more easily than their younger counterparts during functional activities, yet controlled laboratory studies have demonstrated the opposite; muscle becomes more fatigue-resistant with age. We hypothesized that increased task intensities during daily activities due to strength loss may explain this apparent dichotomy. Accordingly, the purpose of this study was to model the potentially opposing influences of age-related improvements in muscle fatigue properties coupled with declining strength on predicted task endurance.
\end{abstract}

Methods: We developed two mathematical models to simulate the relative influences of age-related changes in muscle fatigue and strength: 1) We adapted known endurance time-intensity fatigue models for young adults for older adults based on previously published data; and 2) We modeled changes in functional task intensity for varying levels of strength loss. Using these models, we predicted endurance times for functional tasks requiring from 10$50 \%$ maximum strength.

Results: Even small declines in strength (i.e., 10\%) more than offset these age-related improvements in fatigue resistance when modeling endurance time for functional tasks. For example, a $30 \%$ decline in strength resulted in an approximately $50 \%$ reduction in functional task endurance time, particularly for low intensity tasks.

Conclusions: This study provides a plausible explanation for the apparent dichotomy between laboratory fatigue studies, showing greater fatigue-resistance with advancing age, and anecdotal observations that older adults fatigue more rapidly with advancing age. Our findings suggest that declines in strength will ultimately have a larger effect on fatigue for functional tasks despite the known age-related improvements in fatigue-resistance with age. This information suggests targeted strengthening interventions may be effective in reducing muscle fatigue associated with functional activities, but future controlled trials are needed to validate these findings.

Keywords: Maximum endurance time; Rohmert curves; Aging; Sarcopenia; Dynapenia; Activities of daily living, Muscle fatigue

\section{Introduction}

Sarcopenia [1-3], the age-related loss of muscle mass, and dynapenia, [2,4], the age-related loss of muscle strength, are increasingly recognized as significant adverse detriments often associated with advanced age. Loss of muscle mass and strength increase the risk of physical disability [5], poor quality of life [6] and death [7]. Conversely, numerous studies have demonstrated what may be viewed as a positive adaptation with aging, namely that muscle becomes more fatigue-resistant with advancing age.

Two recent meta-analyses have supported an increase in age-related fatigue resistance based on controlled laboratory conditions; reporting overall moderate effect sizes on the order of 0.49 [8] and 0.56 [9] based on 46 and 37 studies, respectively. These controlled laboratory conditions rely largely on normalized static workloads, i.e., isometric contractions performed at a percentage of each individual's maximum strength, to assess for differences in muscle fatigue properties. Several potential mechanisms have been proposed to explain the observed age-related improvements in fatigue-resistance, such as slower contractile properties that may result from a preferential loss of type II muscle fibers [10-12]; lower motor unit discharge rates [13,14]; and greater reliance on and use of oxidative metabolism [15]. One may assume therefore that age does not result in any decrement in muscle fatigue behavior, and in fact, improves for static contractions.

This improvement in muscle fatigue properties with age seems to be at odds with what is more commonly observed during everyday living, namely that older adults often fatigue more quickly than younger adults during functional physical activities [16]. Similarly, the perception of fatigue is a significant complaint in older adults [17-19] and predicts functional deficits over time including gait speed and physical performance [20]. Avin and Frey Law (2011) found that the age-related fatigue advantage observed with static tasks was not present for dynamic tasks (effect size $=0.05$ ) [8]. However, this still does not explain the greater fatigue observed in older populations during functional tasks. The disconnect between improved endurance 
in laboratory studies and decreased endurance with functional tasks with advanced age has yet to be clearly resolved and in fact is rarely discussed. While strength typically declines with age, the absolute joint torques needed to perform functional tasks or activities of daily living (ADLs) often change minimally with age $[21,22]$. Consequently, many functional tasks involve higher relative task intensities (percentage of maximum strength) with advancing age [21-23]. Because higher intensity contractions cannot be maintained for as long as lower intensity tasks $[8,24]$, if a given task requires a higher proportion of peak strength capability, it follows that fatigue (e.g. endurance time) will occur more quickly. We propose that age-related strength loss, e.g., sarcopenia, and the resulting increase in relative task intensity for any given task (i.e., rising from a chair) with age, may be sufficient to offset the age-related physiological improvements in fatigueresistance. Thus, we hypothesize it is the opposing influences of these two age-related changes that can result in declines in endurance with daily activities despite improvements in muscle fatigue properties.

To test our hypothesis, we have developed a conceptual model linking age-related changes in muscle strength and age-related improvements in muscle fatigue properties to predict endurance behavior during functional tasks, using previously developed mathematical fatigue models and available data in the literature. Thus, the purpose of this study was to examine the relationship between strength declines and muscle fatigue properties in older adults using simulation techniques to better understand the apparent dichotomy between improved muscle fatigue properties seen in laboratory studies and declines in endurance observed functionally with advancing age. This information may be useful in developing optimal rehabilitative strategies to best counteract fatigue during functional tasks in the aging population.

\section{Materials and Methods}

We developed two models for this simulation study. First, we modified previously reported mathematical fatigue models for young adults to represent older adults, referred to as Endurance time (ET) intensity models. Second, we modeled changes in relative task intensity of daily activities due to muscle weakness, referred to as relative intensity-strength decline models. Using these two models cooperatively, we then simulated how long (i.e., endurance time, ET) upper extremity (elbow) and lower extremity (knee) muscles would be able to sustain a contraction needed for a functional task. This enabled us to determine the relative influences of age-related declines in strength and improvements in fatigue-resistance on functional task endurance.

\section{Endurance time (ET)-Intensity models for older adults}

It has been long recognized that how long a muscle contraction can be sustained (i.e. endurance time) is inversely related to the intensity of the task. This relationship has been most fully developed in the ergonomics literature where it is known as the endurance timeintensity relationship or "Rohmert's curve" [24]. Studies have demonstrated that this relationship is nonlinear; muscle contractions can be held exponentially longer as the relative task intensity (\% max) decreases [24-26]. These curves typically represent only static or quasistatic contractions, as they are easily defined, controlled and performed in a laboratory setting. Accordingly, they may best represent slow functional and postural tasks, such as slowly raising from a chair (knee torque) or holding groceries (elbow or shoulder torque). We previously developed endurance time-intensity models for young adults [26] that we modified for this study by increasing the expected endurance times according to mean age-related differences in endurance time observed in the literature. This adaptation would cause a rightward shift in the endurance time- intensity curve for older adults.

The mean effect size used for estimating the age-related fatigue advantage was determined from a previously published meta-analysis [8]. The full details of the age-related fatigue meta-analysis, including inclusion and exclusion criteria, are available elsewhere [8]. The largest age-related fatigue advantage has been observed with static contractions [8]. To examine if strength declines can offset even a relatively large age-related advantage in fatigue resistance and to best match the endurance time-intensity models, we used effect sizes from static contractions only. The effect sizes used to shift our previously reported young adult endurance time models to better represent older adults were Hedge's g=0.61 and 1.08 for the knee and elbow, respectively. Because effect size is a standardized estimate of between group differences, we were able to use these values to estimate the endurance time curves for older adults as a function of task intensity (\% maximum) using the young adult mean and standard deviation estimates [26].

\section{Relative intensity-strength decline models}

With advancing age, the absolute joint torques required to complete a functional task do not appreciably change [21,22], but with advancing age, peak joint torque, a measure of strength, typically declines. Thus the same functional task can require a greater proportion of an older adult's peak strength (i.e., greater relative effort). For example, if holding a bag of groceries or laundry requires $40 \mathrm{Nm}$ of elbow flexion torque and the individual's peak elbow strength is $100 \mathrm{Nm}$, then the task requires $40 \%$ of their maximum strength. If peak strength were to decline by $20 \%$ to $80 \mathrm{Nm}$, that same task (i.e., requiring $40 \mathrm{Nm}$ ) would now represent an intensity of $50 \%$ of maximum (i.e., $40 / 80$ or $50 \%$ ). Mathematically, we modelled this increase in task intensity (referred to as the adjusted task intensity) as a function of strength decline (eq 1); where the ratio, 1/(1-strength decline) acts as a multiplier of the "initial" task intensity (i.e., before the strength decline). For this analysis, five age-related strength declines from $10 \%$ to $50 \%$ were evaluated $(0.1$ to 0.5 , by 0.1 increments), representing the range of age-related strength loss previously observed with aging [2,27-29].

$\%$ Task intensity $=(\%$ Initial Task Intensity $) /(1$-strength decline $)$ (eq. 1)

\section{Combining models to estimate functional endurance time}

Using the two mathematical models described above, we simulated functional task endurance times for both young and old adults, with and without strength loss. We assumed the functional tasks involved quasi-static contractions, such as holding an object with both hands or slowly rising from sit-to-stand. We represented these hypothetical functional tasks simply as a given initial task intensity (i.e., effort expected in young adults) ranging from $10 \%$ to $50 \%$ of maximum effort. This represents a wide range of daily tasks such as holding light to moderately heavy objects, rising slowly from different chair heights with or without upper extremity assistance, or stair climbing.

These initial task intensities (10-50\% max) were used to predict the baseline endurance times for young and older adults using the endurance time-intensity model, allowing for changes in fatigue 
Citation: Keith AG, Aruna T, John LMSE, Frey-Law LA (2015) Strength Loss Counteracts Age-related Improvements in Muscle Fatigue Properties when Considering Functional Task Endurance-A Modeling Study. J Gerontol Geriatr Res 4: 218. doi: $10.4172 / 2167-7182.1000218$

Page 3 of 7

properties with aging without a loss of strength. The same functional task intensities were then adjusted to account for strength loss (e.g., sarcopenia) using the relative strength-intensity model. These adjusted intensities were then applied to the endurance time-intensity model to obtain adjusted ETs for each level of strength loss. Thus, the same functional task was modelled as requiring greater proportions of maximum strength as a function of the muscle strength decline. For example, a functional task requiring $30 \%$ of maximum effort initially would increase to $33.3 \%$ and $60 \%$ of maximum effort with $10 \%$ and $50 \%$ strength losses, respectively. Endurance times for each combination of initial task intensity and degree of strength decline were assessed for older adults. Thus, this methodology allowed us to estimate changes in endurance time for functional tasks between young and older adults considering age-related changes in muscle fatigue properties with and without the confounding effects of agerelated weakness.

\section{Results}

\section{Age-adjusted joint-specific endurance time models}

The old adult endurance time-intensity curves for the knee and elbow shifted to the right by approximately $16 \%$ and $23 \%$, respectively (Figure 1). The corresponding equations relating ET to intensity (0 1.0 range of intensities) were: $\mathrm{ET}=22.043^{*}$ (Intensity $)^{-2.212}$ for the knee and $\mathrm{ET}=22.225^{\star}$ (Intensity $)^{-1.892}$ for the elbow. This represents the frequently reported improvement in fatigue resistance with advancing age.

\section{Relative intensity-strength decline models}

The predicted effect of age-related strength decline on relative task intensity is shown in Figure 2 (and Supplemental materials, Table 1). A $10 \%$ strength loss increases the relative task intensity of any given initial workload or effort by 1.11 times using Equation 1, whereas a loss of $60 \%$ of strength predicts task intensities would increase by 2.5 times. However, when the modified task intensity became greater than $100 \%$ (maximum capability), we applied a maximum of $100 \%$.

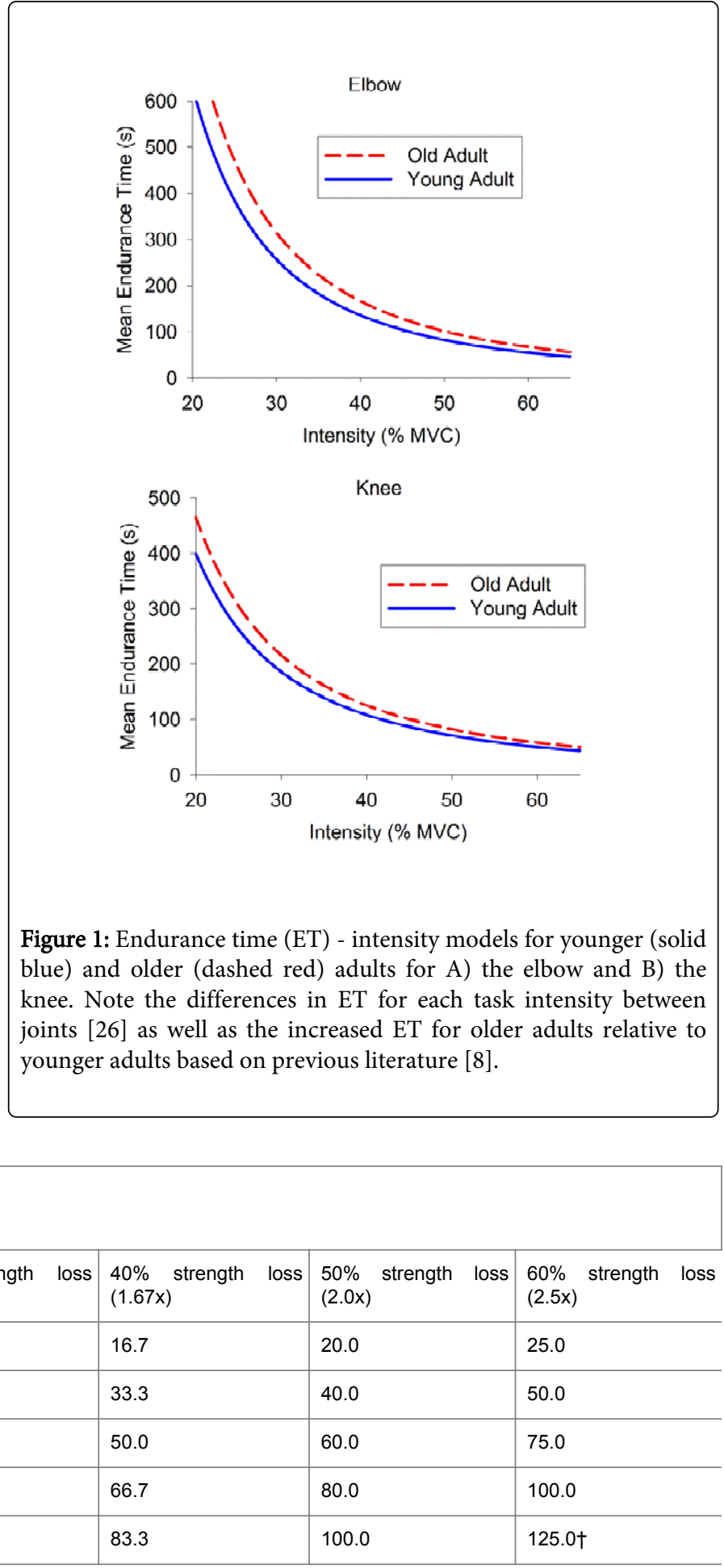

Figure 1: Endurance time (ET) - intensity models for younger (solid blue) and older (dashed red) adults for A) the elbow and B) the Note the differences in ET for each task intensity between oints [26] as well as the increased ET for older adults relative to younger adults based on previous literature [8].

\begin{tabular}{|c|c|c|c|c|c|c|}
\hline \multirow{2}{*}{$\begin{array}{l}\text { Initial Task Intensity } \\
\text { (\% max) }\end{array}$} & \multicolumn{6}{|c|}{$\begin{array}{l}\text { Strength-adjusted Task Intensity } \\
(\% \text { max })\end{array}$} \\
\hline & $\begin{array}{l}10 \% \text { strength loss } \\
(1.11 \mathrm{x})\end{array}$ & $\begin{array}{l}20 \% \text { strength loss } \\
(1.25 x)\end{array}$ & $\begin{array}{l}30 \% \text { strength loss } \\
(1.43 \mathrm{x})\end{array}$ & $\begin{array}{l}40 \% \text { strength loss } \\
(1.67 \mathrm{x})\end{array}$ & $\begin{array}{l}50 \% \text { strength loss } \\
(2.0 \mathrm{x})\end{array}$ & $\begin{array}{l}60 \% \text { strength loss } \\
(2.5 \mathrm{x})\end{array}$ \\
\hline 10.0 & 11.1 & 12.5 & 14.3 & 16.7 & 20.0 & 25.0 \\
\hline 20.0 & 22.2 & 25.0 & 28.6 & 33.3 & 40.0 & 50.0 \\
\hline 30.0 & 33.3 & 37.5 & 42.9 & 50.0 & 60.0 & 75.0 \\
\hline 40.0 & 44.4 & 50.0 & 57.1 & 66.7 & 80.0 & 100.0 \\
\hline 50.0 & 55.6 & 62.5 & 71.4 & 83.3 & 100.0 & $125.0 \dagger$ \\
\hline
\end{tabular}

Table 1: Estimated changes in relative task intensity due to strength declines for tasks requiring initially $10-50 \%$ max intensity. Notes: Adjusted task intensity estimated using Eq 1 from text: Intensity multiplier=1/ (1- relative strength loss). †For values over 100\% (greater than maximal strength capability), a value of 100 was used in all subsequent simulations. 
Citation: Keith AG, Aruna T, John LMSE, Frey-Law LA (2015) Strength Loss Counteracts Age-related Improvements in Muscle Fatigue Properties when Considering Functional Task Endurance-A Modeling Study. J Gerontol Geriatr Res 4: 218. doi: $10.4172 / 2167-7182.1000218$

Page 4 of 7

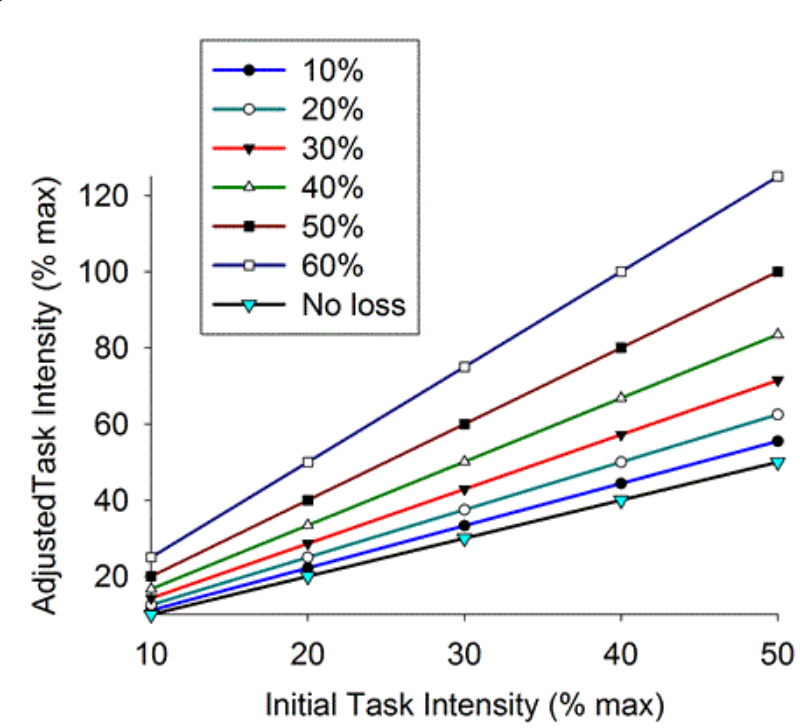

Figure 2: Relative intensity-strength decline models for age-related strength loss ranging from $10-60 \%$. For a given functional task, the initial task intensity represents the \% effort required for a young adult to complete the task. The adjusted task intensity represents the relative effort (\% max) required to complete the functional task as strength declines with aging. Note that no strength loss results in identical adjusted and initial task intensity levels.

\section{Simulating the combined influences of age-related fatigue- resistance and strength loss}

When applying the adjusted relative task intensities to the ETintensity curves for young and older adults, functional task endurance time decreased with each successive increase in strength loss (Table 1). One graphic example is provided in Figure 3, showing the old and young adult endurance time curves for the elbow joint, with three relative task intensities reflecting no strength decline, a $10 \%$ decline and a $30 \%$ decline in strength. When estimating the endurance time for a functional task at the elbow requiring $30 \%$ of maximum intensity, but no strength loss, the endurance time was just over $250 \mathrm{sec}$ for young adults and over $300 \mathrm{sec}$ for older adults without strength loss due to the improved fatigue-resistant muscle properties seen with advanced age (Table 1). However, even a small 10\% loss of strength counteracted the age-related improvement in fatigue resistance (ET declined to $251 \mathrm{sec}$ ), and was further compromised when considering the $30 \%$ decline in strength (ET declined to $143 \mathrm{sec}$ ). This same finding was consistently modelled across initial baseline task intensities and for both knee and elbow joint regions (Table 2). Note, however, the magnitude of the effect is dependent on the initial baseline task intensity, where less strenuous tasks show the most dramatic loss of endurance time with strength loss due to the nonlinear curvature of the endurance time-intensity relationship.

\begin{tabular}{|c|c|c|c|c|c|c|c|c|c|}
\hline \multirow[t]{2}{*}{ Joint } & \multirow{2}{*}{$\begin{array}{l}\text { Initial } \\
\text { Task Intensity } \\
\text { (\% max) }\end{array}$} & \multirow{2}{*}{$\begin{array}{l}\begin{array}{l}\text { Young } \\
\text { Adult ET } \\
\text { (sec)* }\end{array} \\
\text { Full } \\
\text { Strength }\end{array}$} & \multicolumn{7}{|c|}{$\begin{array}{l}\text { Old Adult ET } \\
(\mathrm{sec}) \dagger\end{array}$} \\
\hline & & & $\begin{array}{l}\text { Full } \\
\text { Strength }\end{array}$ & $\begin{array}{l}10 \% \\
\text { loss }\end{array}$ & $\begin{array}{l}20 \% \\
\text { loss }\end{array}$ & $\begin{array}{l}30 \% \\
\text { loss }\end{array}$ & $\begin{array}{l}40 \% \\
\text { loss }\end{array}$ & $\begin{array}{l}50 \% \\
\text { loss }\end{array}$ & $\begin{array}{l}60 \% \\
\text { loss }\end{array}$ \\
\hline \multirow[t]{6}{*}{ Elbow/ } & 10 & 2916 & 3591 & 2851 & 2192 & 1628 & 1155 & 775 & 473 \\
\hline & 20 & 630 & 775 & 615 & 473 & 351 & 249 & 167 & 102 \\
\hline & 30 & 257 & 316 & 251 & 193 & 143 & 102 & 68 & 42 \\
\hline & 40 & 136 & 167 & 133 & 102 & 76 & 54 & 36 & 22 \\
\hline & 50 & 83 & 102 & 81 & 62 & 46 & 33 & 22 & 22 \\
\hline & \multicolumn{2}{|c|}{ Mean\% difference } & $22.9 \%$ & $-2.4 \%$ & $-25.0 \%$ & $-44.3 \%$ & $-60.5 \%$ & $-73.5 \%$ & $-81.7 \%$ \\
\hline \multirow[t]{6}{*}{ Knee } & 10 & 1470 & 1736 & 1425 & 1138 & 882 & 658 & 468 & 307 \\
\hline & 20 & 399 & 468 & 384 & 307 & 238 & 177 & 126 & 83 \\
\hline & 30 & 186 & 217 & 178 & 142 & 110 & 82 & 59 & 38 \\
\hline & 40 & 109 & 126 & 103 & 83 & 64 & 48 & 34 & 22 \\
\hline & 50 & 71 & 83 & 68 & 54 & 42 & 31 & 22 & 22 \\
\hline & \multicolumn{2}{|c|}{ Mean \% differenceł } & $16.5 \%$ & $-4.2 \%$ & $-23.5 \%$ & $-40.7 \%$ & $-55.8 \%$ & $-68.6 \%$ & $-77.2 \%$ \\
\hline
\end{tabular}

Table 2: Predicted endurance times (ET) for young and old adults (with and without strength loss) for tasks involving the elbow and knee joints. 
Citation: Keith AG, Aruna T, John LMSE, Frey-Law LA (2015) Strength Loss Counteracts Age-related Improvements in Muscle Fatigue Properties when Considering Functional Task Endurance-A Modeling Study. J Gerontol Geriatr Res 4: 218. doi: $10.4172 / 2167-7182.1000218$

Page 5 of 7

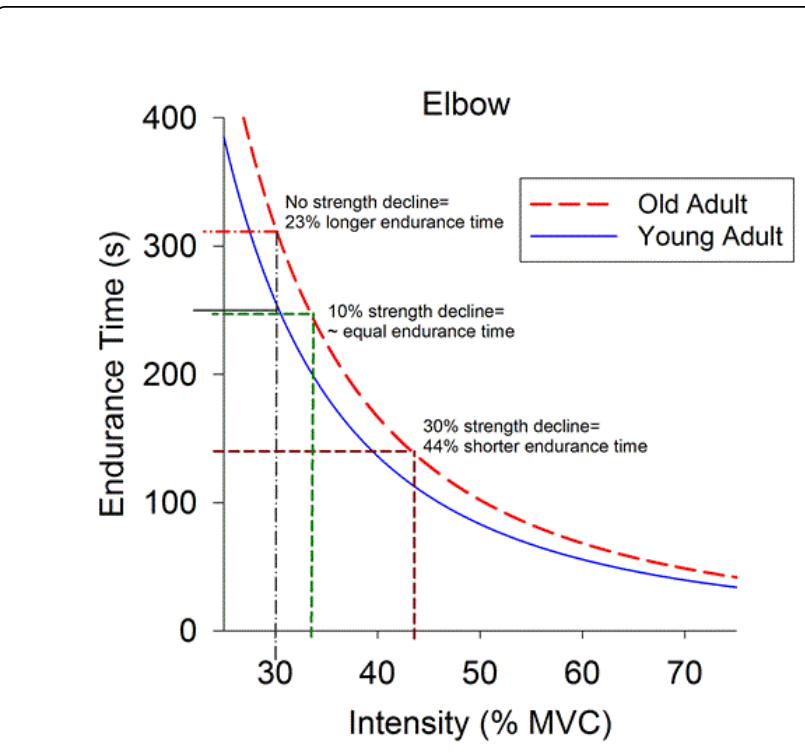

Figure 3: This is an example of applying the strength decline estimates (i.e., increased functional task intensity due to strength loss) to the endurance time (ET) - intensity models. ETs for a functional task requiring 30\% max initial intensity (dot-dash dark gray line) demonstrate the age-related advantage ( 23\%) for the older versus younger adults in the absence of strength decline. However, even a $10 \%$ loss of strength (relative task intensity increases to $33.3 \% \max$ ) offsets this advantage which worsens with greater strength declines (i.e., only $10 \%$ and $30 \%$ decline in strength are shown).

\section{Discussion}

The main purpose of this study was to assess age-related changes in functional task endurance considering both enhanced muscle fatigueresistance properties and varying levels of strength loss using simulations and known mathematical relationships of muscle fatigue. In particular, this study found even a small degree of strength loss (e.g., 10\%) more than offsets the average improvement in age-related fatigue resistance reported previously $[8,9]$. These findings provide a plausible explanation for the apparent dichotomy between greater fatigue with aging for functional tasks despite improved muscle fatigue resistance when assessed under controlled, relative-intensity laboratory conditions. Further, these findings suggest that rehabilitative efforts targeting strength gain (or even maintenance) may provide significant improvements in functional task endurance, particularly for low intensity tasks. However, the extent to which loss of strength impairs function or the interplay between atrophy and strength were not extrapolated from the current model. Rather, this model identified age-related strength loss and associated change in the ability to perform a functional task over time.

Localized muscle fatigue may depend on several factors, such as variations in fiber type [30], motor unit distribution/activation [31], neural activation [32], task specificity [33], and/or absolute force/ muscle cross-sectional area [34]. The complex interplay of these processes may be further influenced by common age-related changes in human muscle, including: muscle fiber atrophy [10], reduced number of motor units [35], contractile slowing [36] and a transition towards slow-twitch muscle fibers [37]. Although, it is not clear how these age-related changes proportionally influence fatigue development, it is clear that the net fatigue response is altered with aging. This alteration allows for older adults to maintain a given relative-intensity contraction longer than young adults as represented by a rightward shift in the endurance time - intensity (i.e., "Rohmert") curves.

While significant differences in endurance time exist with advancing age for relative-intensity tasks, as demonstrated by significant moderate to large effect sizes consistently seen in the literature $[8,9]$, these age-related differences produced relatively small percent changes in endurance time. For example, we have previously demonstrated the magnitude of age-related differences in ET vary between young and old adults from 10-23\% [8]. While these agerelated improvements in fatigue resistance have been reliably observed for isometric contractions $[8,9]$, in our modeling study, they were readily offset by even small losses of strength.

Studies evaluating joint torques for functional activities between younger and older adults are consistent with our findings, providing a degree of construct validity of our models. Analyses of sit-to-stand transfers and stair climbing indicate relative task intensities of these functional tasks are greater in older versus young adults [22,23], but no differences with age have also been reported [38]. When differences have been observed, tasks required $30-54 \%$ of maximum strength for the young adult and $78-100 \%$ of maximum in older adults [22,23]. This is despite nearly identical or even higher absolute knee joint loads in the young cohorts to perform the sit-to-stand task compared to the older aged cohorts [21-23]. These increases in task intensity for functional tasks with aging are consistent with our relative intensitystrength decline model. We estimated tasks of $30-50 \%$ maximum effort in young adults would increase to $43 \%$ to $71 \%$ maximum effort in older adults experiencing a 30\% loss of strength. Accordingly, these previous biomechanical analyses support our estimates of increased relative task intensities as a function of age-related muscle weakness for functional tasks. Currently, among the multiple professional organizations, societies and workgroups, there is not a consensus on how to best define sarcopenia clinically. While sarcopenia involves loss of muscle mass, declines in peak strength (also referred to as dynapenia), performance and function assessments may also provide useful measures of sarcopenia. While this study broadly focused on the commonly observed levels of strength loss with aging; this loss may occur as the result of atrophy or impaired muscle quality that may or may not be clinically defined as sarcopenia.

Anecdotally, it is common to think of older adults as fatiguing more rapidly, which is consistent with the finding that older adults fatigue more quickly or to a greater extent than their younger counterparts for functional tasks [16]. Interestingly, this same relationship between the development of muscle fatigue during relative and absolute task intensities was noted several decades ago, distinct from aging effects [39]. Stronger and weaker young men were equally able to sustain a relative task intensity ( $62.5 \% \max )$, but when using an absolute force (50 lbs) the weakest men fatigued more quickly than the strongest men. While the explanation for this is likely multifactorial including muscle perfusion, glycolytic versus oxidative energy utilization, etc., similar behavior is explained in our study using the traditional "Rohmert" curves and adjustments for declines in peak strength, despite similar absolute joint torques (i.e., elevated relative task intensity as a result of reduced peak strength). 
Citation: Keith AG, Aruna T, John LMSE, Frey-Law LA (2015) Strength Loss Counteracts Age-related Improvements in Muscle Fatigue Properties when Considering Functional Task Endurance-A Modeling Study. J Gerontol Geriatr Res 4: 218. doi: $10.4172 / 2167-7182.1000218$

Page 6 of 7

Due to the nonlinear relationship between relative task intensity and endurance time, these results demonstrate that even moderate reductions in strength loss could result in relatively large improvements in functional task endurance time. This was particularly true for tasks that require low relative intensities prior to strength loss. For example, if an older individual were to exhibit a strength loss of approximately $40 \%$ compared to young adult levels, rehabilitation exercises resulting in a net loss of only $30 \%$ compared to young adults would increase the endurance time for a low-intensity functional task (i.e., $10 \%$ of maximum) by 35 to $40 \%$. Accordingly, these results suggest that strength training may provide a desirable approach for therapeutic interventions for older adults not only for the purpose of improving strength, but also for improving daily activity endurance, given the inter-connectedness between strength and fatigue for functional tasks. Further, this information may have an impact when evaluating the clinically utility of power versus strength training in older adult. Focusing on strength training will not only increase strength, but improve the ability to repeatedly perform a functional task. Future investigations should consider the impact strength training versus power training may have upon endurance, functional performance and falls.

A limitation of this current study is that we did not include the many additional factors that likely change with advancing age, including body mass or anthropometric distribution of mass, and did not explicitly model age-related differences such as muscle fiber type or motor unit activation ratio. Clearly, this investigation employed a heuristic model that simply represents the global behavioral changes observed in younger versus older adult cohorts, namely improved fatigue-resistance properties coupled with declines in peak muscle strength. Further, we recognize peak strength is not an isolated influence on the relative task intensity for functional tasks, but for these analyses we made the assumption that all other biomechanical factors remained constant (i.e. body weight and limb lengths). This allowed us to focus on the relative influences of strength loss versus improvements in muscle fatigue properties with advancing age. Thus, our models reflect the differences that might occur between "matched" pairs of individuals (i.e. similarly sized) or the same individual across time (e.g. considering changes in strength and fatigue resistance only). Despite their simplicity, these models highlight the relative importance of using age-appropriate model inputs (i.e. relative task intensity for a functional task) when quantifying fatigue differences (e.g. time to task failure or endurance time) for young and old adults.

In summary, this study provides a plausible explanation for the apparent dichotomy between laboratory fatigue studies using relativeintensity tasks, showing greater fatigue-resistance with advancing age, and the more commonly held anecdotal observations that older adults fatigue more rapidly with advancing age. Our findings suggest that declines in strength will ultimately have a larger effect on fatigue for functional tasks despite the known age-related improvements in fatigue-resistance with age. This information suggests targeted strengthening interventions may be effective in reducing muscle fatigue associated with functional activities, but future controlled trials are needed to validate these findings.

\section{References}

1. Narici MV, Maffulli N (2010) Sarcopenia: characteristics, mechanisms and functional significance. British medical bulletin 95: 139-159.

2. Mitchell WK, Williams J, Atherton P, Larvin M, Lund J, et al. (2012) Sarcopenia, dynapenia, and the impact of advancing age on human skeletal muscle size and strength; a quantitative review. Frontiers in physiology 3: 260.

3. Cruz-Jentoft AJ, Baeyens JP, Bauer JM, Boirie Y, Cederholm T, et al. (2010) Sarcopenia: European consensus on definition and diagnosis: Report of the European Working Group on Sarcopenia in Older People. Age Ageing 39: 412-423.

4. Manini TM, Clark BC (2012) Dynapenia and aging: an update. The journals of gerontology Series A, Biological sciences and medical sciences 67: 28-40.

5. Janssen I, Heymsfield SB, Ross R (2002) Low relative skeletal muscle mass (sarcopenia) in older persons is associated with functional impairment and physical disability. Journal of the American Geriatrics Society 50: 889-896.

6. Go SW, Cha YH, Lee JA, Park HS (2013) Association between sarcopenia, bone density, and health-related quality of life in Korean men. Korean J Fam Med 34: 281-288.

7. Newman AB, Kupelian V, Visser M, Simonsick EM, Goodpaster BH, et al. (2006) Strength, but not muscle mass, is associated with mortality in the health, aging and body composition study cohort. The journals of gerontology Series A, Biological sciences and medical sciences 61: 72-77.

8. Avin KG, Frey Law LA (2011) Age-related differences in muscle fatigue vary by contraction type: a meta-analysis. Phys Ther 91: 1153-1165.

9. Christie A, Snook EM, Kent-Braun JA (2011) Systematic review and meta-analysis of skeletal muscle fatigue in old age. Med Sci Sport Exer 43: 568-577.

10. Lexell J, Taylor CC (1991) Variability in muscle-fiber areas in whole human quadriceps muscle - effects of increasing age. J Anat 174: 239-249.

11. Lexell J (1995) Human aging, muscle mass, and fiber type composition. The journals of gerontology Series A, Biological sciences and medical sciences 50 Spec No: 11-16.

12. Brunner F, Schmid A, Sheikhzadeh A, Nordin M, Yoon J, et al. (2007) Effects of aging on Type II muscle fibers: a systematic review of the literature. Journal of aging and physical activity 15: 336-348.

13. Rubinstein S, Kamen G (2005) Decreases in motor unit firing rate during sustained maximal-effort contractions in young and older adults. Journal of electromyography and kinesiology: official journal of the International Society of Electrophysiological Kinesiology 15: 536-543.

14. Kamen G, Sison SV, Du CC, Patten C (1995) Motor unit discharge behavior in older adults during maximal-effort contractions. Journal of applied physiology 79: 1908-1913.

15. Lanza IR, Befroy DE, Kent-Braun JA (2005) Age-related changes in ATPproducing pathways in human skeletal muscle in vivo. Journal of applied physiology 99: 1736-1744.

16. Nakao M, Inoue Y, Murakami H (1989) Aging process of leg muscle endurance in males and females. European journal of applied physiology and occupational physiology 59: 209-214.

17. Leveille SG, Fried LP, McMullen W, Guralnik JM (2004) Advancing the taxonomy of disability in older adults. The journals of gerontology Series A, Biological sciences and medical sciences 59: 86-93.

18. Gill TM, Desai MM, Gahbauer EA, Holford TR, Williams CS (2001) Restricted activity among community-living older persons: Incidence, precipitants, and health care utilization. Ann Intern Med 135: 313-321.

19. Eldadah BA (2010) Fatigue and fatigability in older adults. PM\&R 2: 406-413.

20. Hardy SE, Studenski SA (2008) Fatigue and function over 3 years among older adults. The journals of gerontology Series A, Biological sciences and medical sciences 63: 1389-1392.

21. Schultz AB, Alexander NB, Ashtonmiller JA (1992) Biomechanical analyses of rising from a chair. J Biomech 25: 1383-1391.

22. Hughes MA, Myers BS, Schenkman ML (1996) The role of strength in rising from a chair in the functionally impaired elderly. J Biomech 29: 1509-1513.

23. Hortobagyi T, Mizelle C, Beam S, DeVita P (2003) Old adults perform activities of daily living near their maximal capabilities. The journals of 
Citation: Keith AG, Aruna T, John LMSE, Frey-Law LA (2015) Strength Loss Counteracts Age-related Improvements in Muscle Fatigue Properties when Considering Functional Task Endurance-A Modeling Study. J Gerontol Geriatr Res 4: 218. doi: $10.4172 / 2167-7182.1000218$

Page 7 of 7

gerontology Series A, Biological sciences and medical sciences 58 M453-460.

24. Rohmert W (1960) Ermittlung von erholungspausen für statische arbeit des menschen. Int $Z$ angew Physiol einschl Arbeitsphysiol 18: 123-164.

25. El ahrache K, Imbeau D, Farbos B (2006) Percentile values for determining maximum endurance times for static muscular work. Int $J$ Ind Ergon 36: 99-108.

26. Frey Law LA, Avin KG (2010) Endurance time is joint-specific: a modelling and meta-analysis investigation. Ergonomics 53: 109-129.

27. Hortobagyi T, Zheng D, Weidner M, Lambert NJ, Westbrook S, et al. (1995) The influence of aging on muscle strength and muscle fiber characteristics with special reference to eccentric strength. The journals of gerontology Series A, Biological sciences and medical sciences 50: B399-406.

28. Hughes RE, Johnson ME, O'Driscoll SW, An KN (1999) Age-related changes in normal isometric shoulder strength. Am J Sport Med 27: 651-657.

29. Stoll T, Huber E, Seifert B, Michel BA, Stucki G (2000) Maximal isometric muscle strength: normative values and gender-specific relation to age. Clinical rheumatology 19: 105-113.

30. Burke RE, Levine DN, Tsairis P, Zajac FE (1973) Physiological types and histochemical profiles in motor units of the cat gastrocnemius. The Journal of physiology 234: 723-748.

31. Bigland-Ritchie B, Woods JJ (1984) Changes in muscle contractile properties and neural control during human muscular fatigue. Muscle \& nerve 7: 691-699.
32. Clark BC, Collier SR, Manini TM, Ploutz-Snyder LL (2005) Sex differences in muscle fatigability and activation patterns of the human quadriceps femoris. European journal of applied physiology 94: 196-206.

33. Hunter SK, Rochette L, Critchlow A, Enoka RM (2005) Time to task failure differs with load type when old adults perform a submaximal fatiguing contraction. Muscle \& nerve 31: 730-740.

34. Hunter SK, Enoka RM (2001) Sex differences in the fatigability of arm muscles depends on absolute force during isometric contractions. Journal of applied physiology 91: 2686-2694.

35. Brown WF (1972) A method for estimating the number of motor units in thenar muscles and the changes in motor unit count with ageing. Journal of neurology, neurosurgery, and psychiatry 35: 845-852.

36. Doherty TJ, Brown WF (1997) Age-related changes in the twitch contractile properties of human thenar motor units. Journal of applied physiology 82: 93-101.

37. Larsson L, Sjodin B, Karlsson J (1978) Histochemical and biochemical changes in human skeletal muscle with age in sedentary males, age 22--65 years. Acta physiologica Scandinavica 103: 31-39.

38. Bieryla KA, Anderson DE, Madigan ML (2009) Estimations of relative effort during sit-to-stand increase when accounting for variations in maximum voluntary torque with joint angle and angular velocity. Journal of electromyography and kinesiology: official journal of the International Society of Electrophysiological Kinesiology 19: 139-144.

39. Start KB, Graham JS (1964) Relationship between the relative and absolute isometric endurance of an isolated muscle group. Res Quart 35: 193-204. 